Supporting Information

\title{
Investigations of Molecular Optical Properties Using Quantum Light and Hong-Ou-Mandel Interferometry
}

Audrey Eshun ${ }^{\ddagger}$; Bing Gu $u^{\S}$; Oleg Varnavski ${ }^{\ddagger}$,Shahaf Asban ${ }^{\S}$; Konstantin E. Dorfman ${ }^{\dagger}$; Shaul Mukamel ${ }^{\S}$; Theodore Goodson III *** $^{*}$

\section{Polarization Visibility Test}

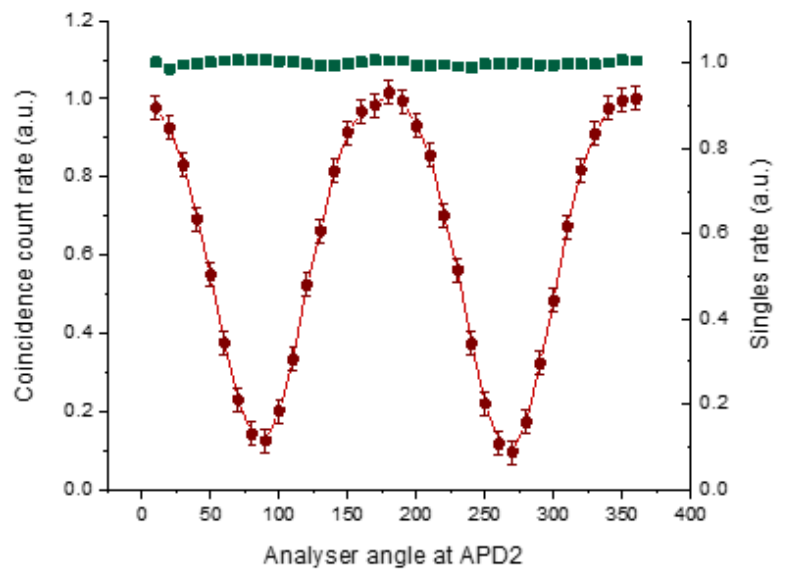

Figure S1. Graph showing polarization visibility. Normalized coincidence count rate as a function of polarizer angle (red). Singles count rate as a function of polarizer angle (green).

A polarization visibility test was conducted to determine the degree of polarization entanglement of the SPDC light to ensure that entanglement is high enough for indistinguishability to be seen in the HOM measurements. In this test, the polarization analyzer at one detector is kept constant while the other analyzer is adjusted, and coincidence counts are measured as a function of this polarizer angle, showing where there is maximum entanglement. When the polarizer angle at detector $\mathrm{A}$ is orthogonal to that at detector $\mathrm{B}$, the maximum coincidence counts are observed, confirming the polarization entanglement of the photon pairs. Alternatively, when the polarization angles are the same, no coincidences are 
detected since detector A does not register the twin photon of the entangled photon pair. Figure S1 shows a visibility of $90 \pm 2 \%$.

\section{$\underline{\text { Materials }}$}

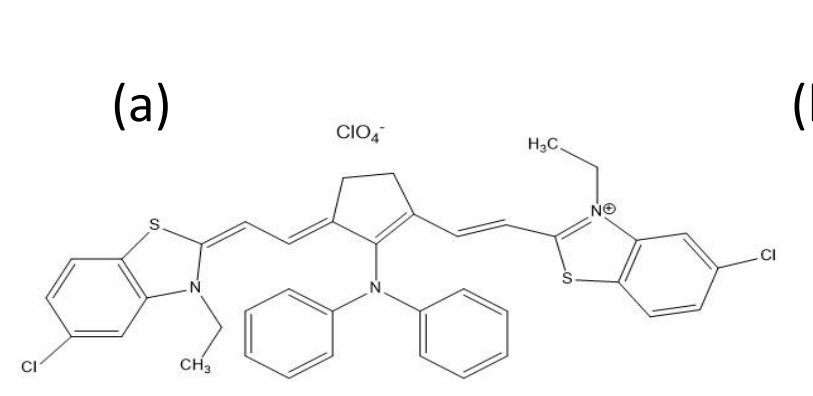

(b)

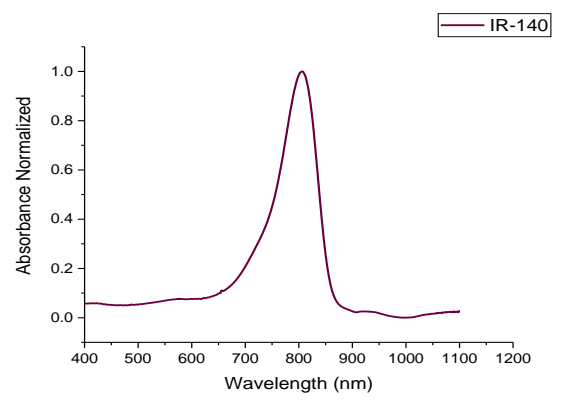

(c)

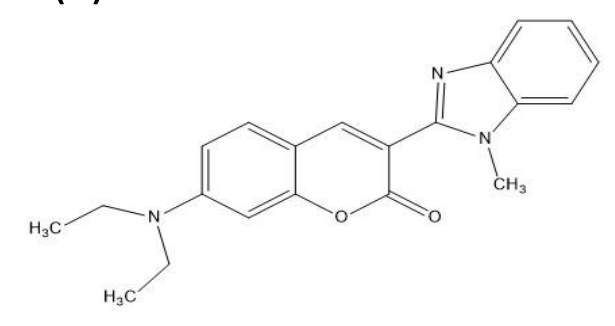

(d)

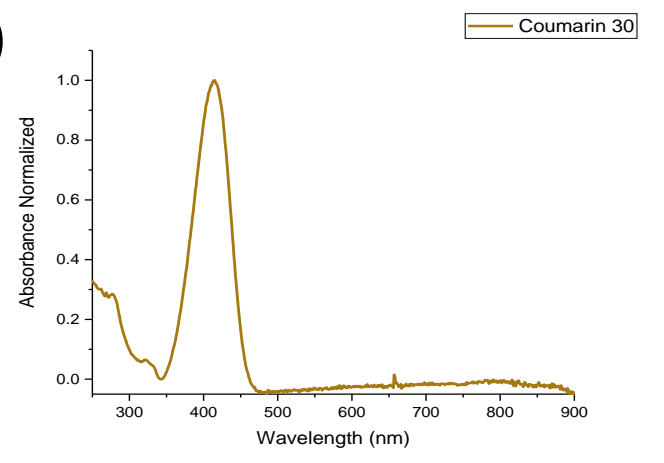

Figure S2. (a) Molecular structure of IR-140. (a) Normalized absorption spectra of IR-140 dye.

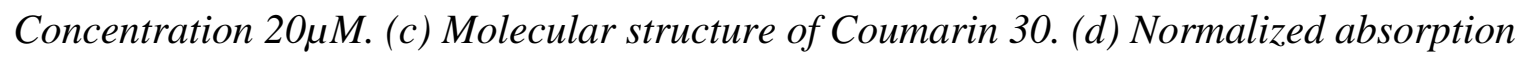
spectra of Coumarin 30 dye. Concentration $29 \mu \mathrm{M}$. 


\section{Simulated Visibility Depending on Pump Bandwidth}

(a)

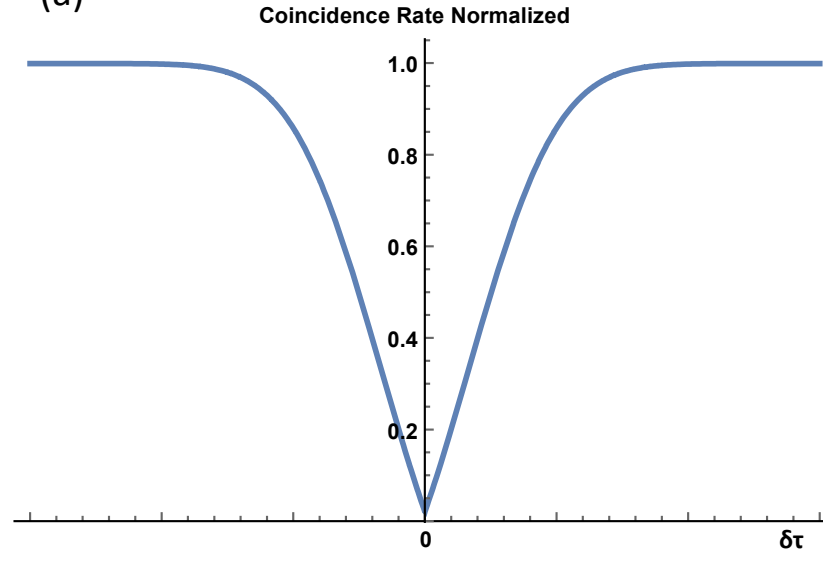

(b)

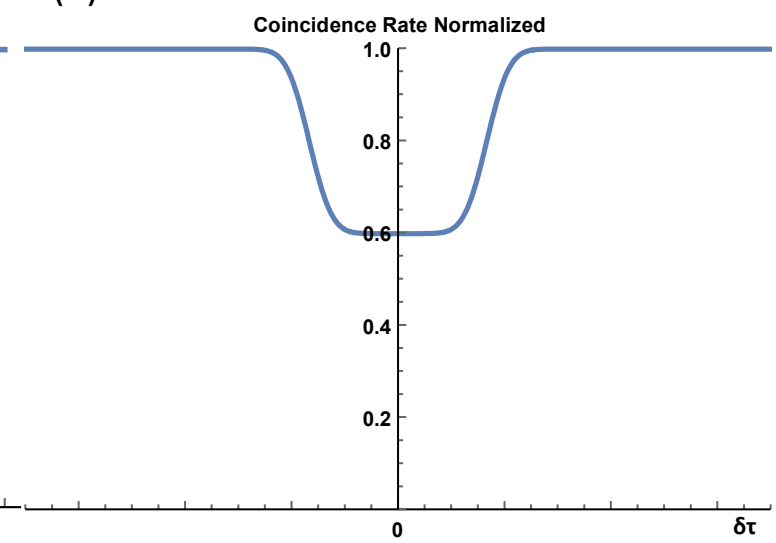

Figure S3. Normalized coincidence rate as a function of time estimated using Eq. 2 for (a) a narrow CW pump bandwidth and (b) a larger femtosecond pump that is $8 \sigma_{C W}$.

\section{HOM Scans with Interference Filter Dependence}

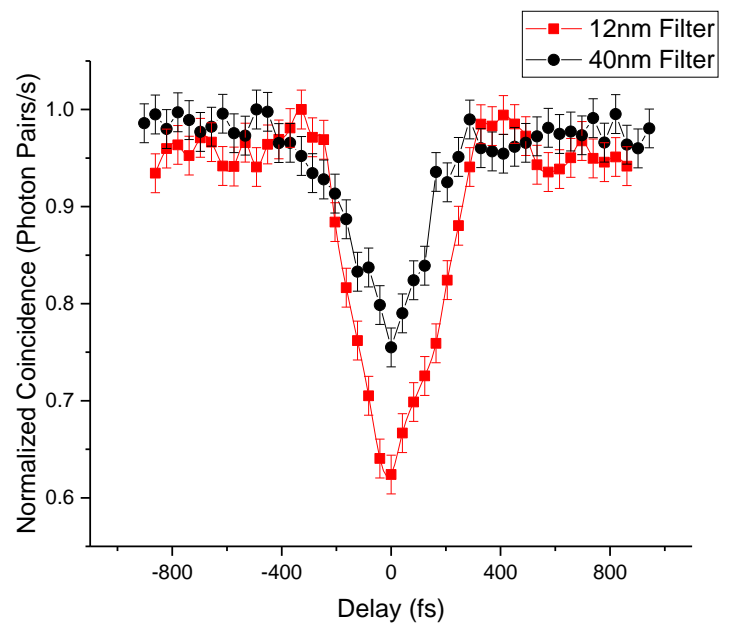

Figure S4. HOM dips measured using $12 \mathrm{~nm}$ interference filter (red) and 40nm interference filter (black). 


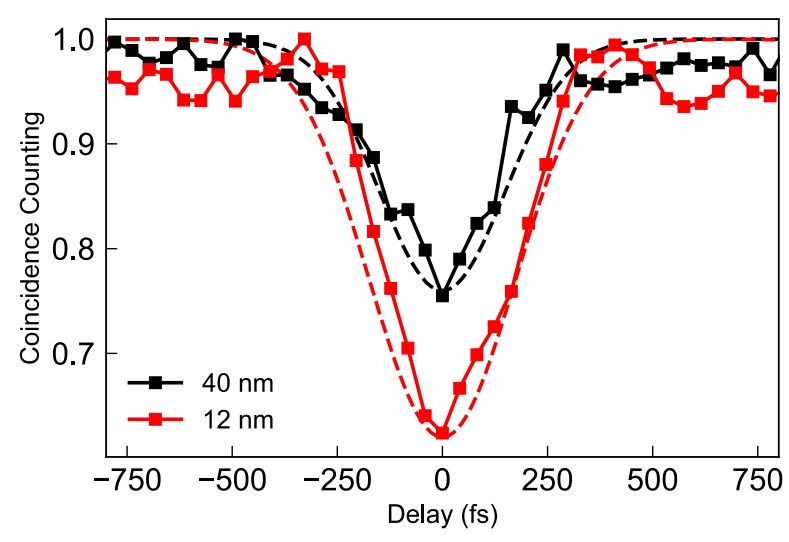

Figure S5. Theoretically fitted HOM dips modelled with different joint spectral amplitudes which include the effects of interference filters.

\section{HOM Scans with Coumarin 30}

The HOM dip with solvent (methanol) placed in the interferometer path is measured and the normalized coincidence rate as a function of time delay is shown in supplementary Fig. S6. Similar to the dip in Figure 3 of the main paper, the dip with solvent is symmetric and triangular, with steadily oscillating counts on both ends before counts drop, signifying the zero-delay position. The declining portion of the dip is smooth on both sides with no oscillations and any changes in amplitude as low as $1 \%$. This reproduced symmetrical triangular shape confirms that the methanol does not absorb the photons or interfere with them and is not excited by them. Thus, methanol does not affect the two-photon quantum interference, and this serves as an accurate control scan.

Subsequently, the pure solvent in the interferometer arm was replaced with Coumarin 30 (dissolved in methanol). As Coumarin 30 has no absorption at $800 \mathrm{~nm}$, this was used to determine how the HOM dip would be affected by a non-resonant medium placed in the interferometer path. There was little to no change with the Coumarin 30 sample placed in the interferometer path (Fig. S6). The HOM dip maintains its symmetric triangular shape, with steady coincidence counts 
oscillating at $\pm 2.5 \%$ on the ends before smoothly descending to the zero-delay position. In addition to the HOM dip maintaining its shape, the coincidence counts from the measurement with Coumarin 30 remain the same and display no attenuation compared to the counts with pure solvent. This indicates that the photons propagated through the non-resonant sample were not absorbed and did not interfere with the sample, thus there was no coherent excitation of the Coumarin 30 chromophore. The photons propagating through this dye experienced no new phenomena or phase change compared to the photons in the opposite path, therefore, upon recombining at the beam splitter there is no difference in their interaction and the HOM dip is unchanged. This was conducted with both 12 and $40 \mathrm{~nm}$ filters to ensure that the broader filter did not result in a different outcome.

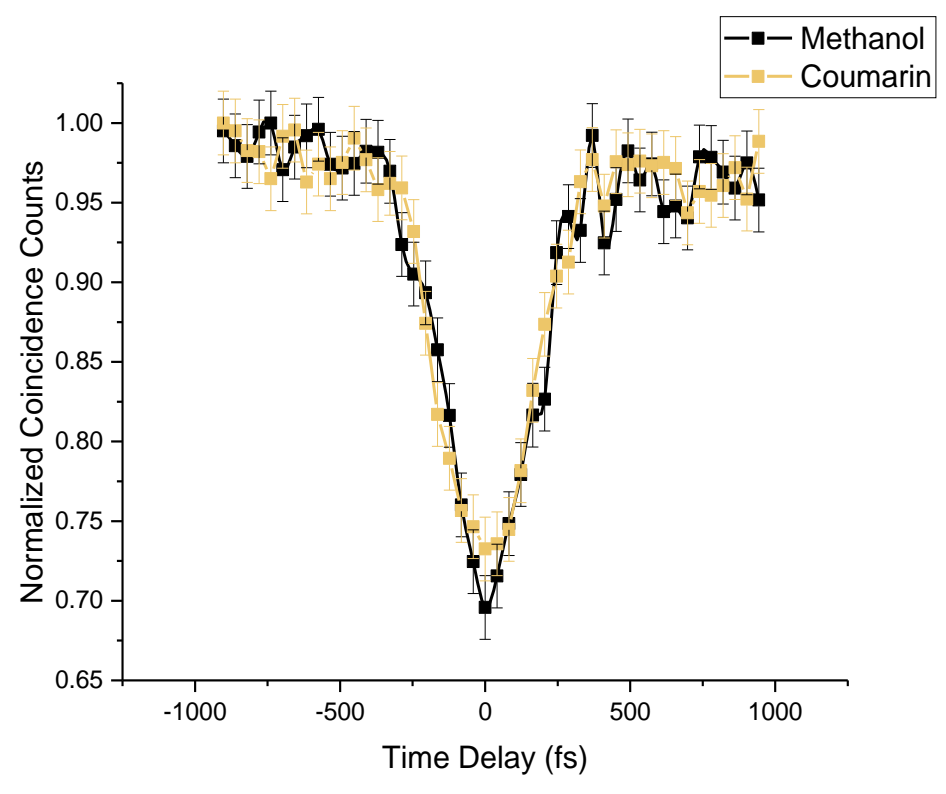

Figure S6. Normalized coincidence counts as a function of time delay showing the HOM dip with a solvent, Methanol, (shown in black) and with a non-resonant sample, Coumarin 30 (shown in yellow). 


\section{$\underline{\text { IR-140 linear Susceptibility }}$}

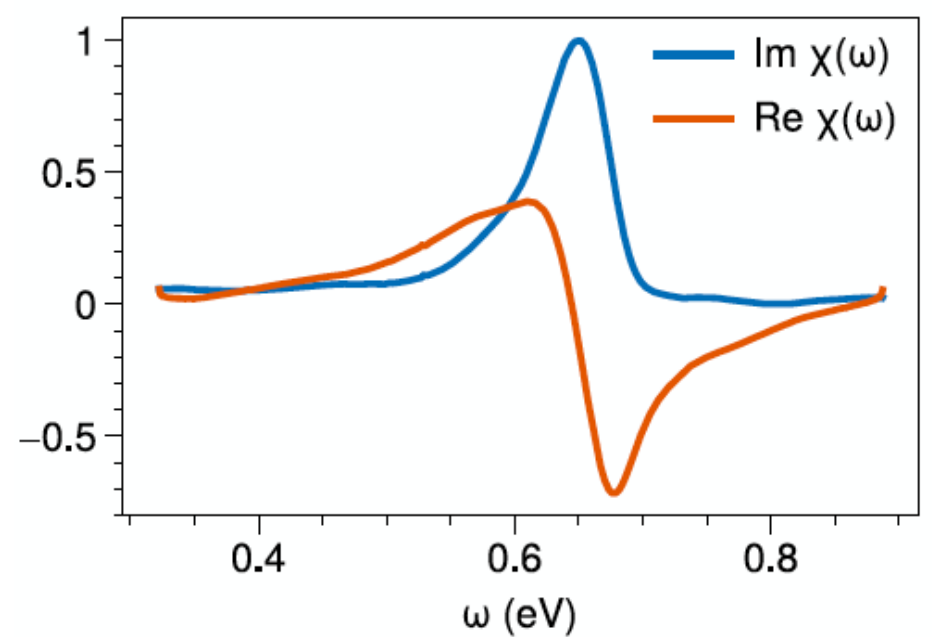

Figure S7. Imaginary, Im $\chi(\omega)$, and real, Re $\chi(\omega)$, parts of the linear susceptibility of the IR-140 molecule The real part is obtained by Hilbert transform of the absorption data.

\section{Theoretical Modelling Overlaid}

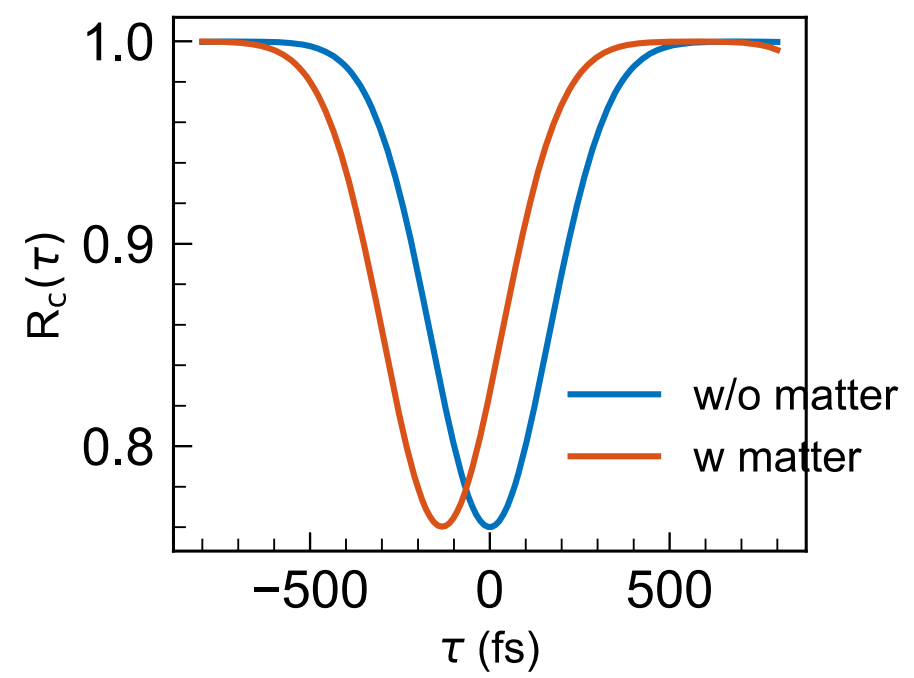

Figure S8. Theoretical modelling of the HOM dips with and without sample overlaid.

\section{Narrowband pump and broadband twin photons}

In the limit of a narrowband pump $\left(\sigma_{+} \rightarrow 0\right)$ and broadband twin photons (i.e. $\sigma_{-}$is larger than 
the characteristic linewidth $\gamma$ of the transmission function), Fourier transform of the HOM coincidence counting rate (Eq. 2) leads to

$R(\tau)=-\frac{1}{2} \int d \Delta \beta^{*}(\Delta) \beta(-\Delta) T^{*}\left(\bar{\omega}-\frac{\Delta}{2}\right) T\left(\bar{\omega}+\frac{\Delta}{2}\right) e^{i \Delta \tau}=-\frac{1}{2} \int d \Delta T^{*}\left(\bar{\omega}-\frac{\Delta}{2}\right) T(\bar{\omega}+$

$\left.\frac{\Delta}{2}\right) e^{i \Delta \tau} \equiv-\frac{1}{2} T_{W}\left(\frac{\omega_{p}}{2}, \tau\right)$

Eq. S1 shows that in this limit, the coincidence counting rate is determined by the Wigner transform of the transmission function. By scanning the pump frequency, it is possible to obtain the full $T_{W}(\omega, \tau)$. Note that the condition $\sigma_{-} \gg \gamma$ is not satisfied in the current experiment.

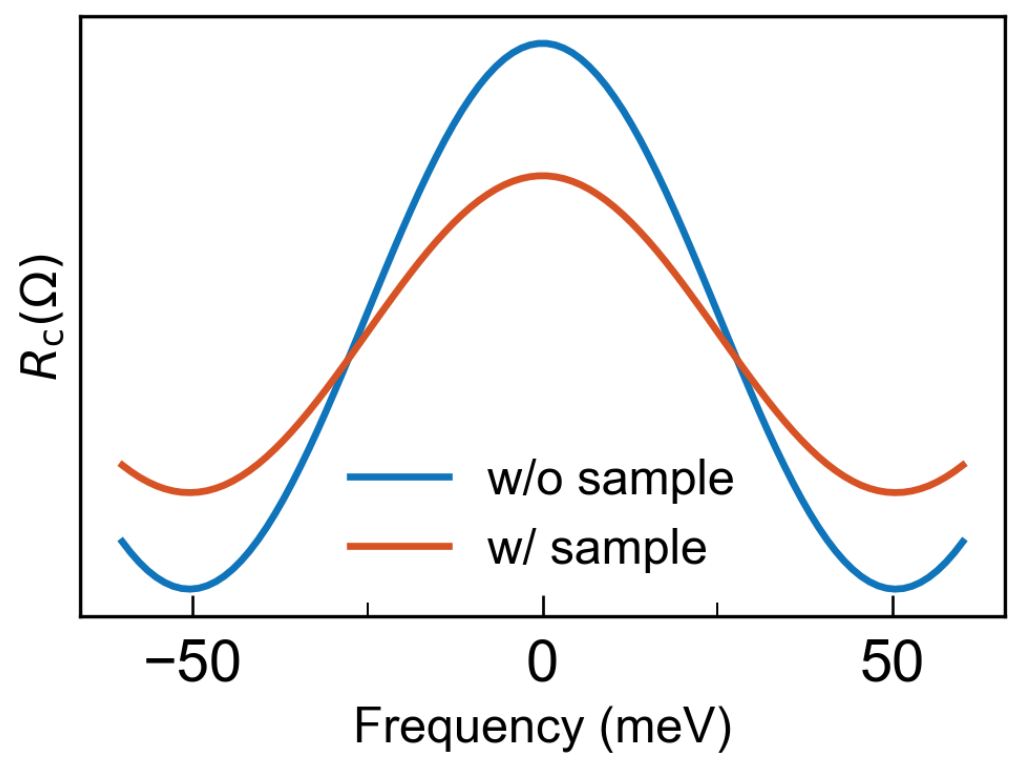

Figure S8. Fourier transform of coincidence rates according to Eq. 8. We observe a difference in the frequency distributions and amplitude for the data with and without a sample. 